\title{
Molecular analysis of clinical isolates previously diagnosed as Mycobacterium intracellulare reveals incidental findings of "Mycobacterium indicus pranii" genotypes in human lung infection
}

Su-Young Kim ${ }^{1 \dagger}$, Hye Yun Park ${ }^{1 \dagger}$, Byeong-Ho Jeong ${ }^{1}$, Kyeongman Jeon ${ }^{1}$, Hee Jae Huh², Chang-Seok Ki ${ }^{2}$, Nam Yong Lee ${ }^{2}$, Seung-Jung Han ${ }^{3}$, Sung Jae Shin ${ }^{3^{*}}$ and Won-Jung Koh ${ }^{1^{*}}$

\section{Abstract}

Background: Mycobacterium intracellulare is a major cause of Mycobacterium avium complex lung disease in many countries. Molecular studies have revealed several new Mycobacteria species that are closely related to $M$. intracellulare. The aim of this study was to re-identify and characterize clinical isolates from patients previously diagnosed with $M$. intracellulare lung disease at the molecular level.

Methods: Mycobacterial isolates from 77 patients, initially diagnosed with $M$. intracellulare lung disease were re-analyzed by multi-locus sequencing and pattern of insertion sequences.

Results: Among the 77 isolates, 74 (96 \%) isolates were designated as M. intracellulare based on multigene sequence-based analysis. Interestingly, the three remaining strains (4\%) were re-identified as "Mycobacterium indicus pranii" according to distinct molecular phylogenetic positions in rpoB and hsp65 sequence-based typing. In hsp65 sequevar analysis, code 13 was found in the majority of cases and three unreported codes were identified. In 16S-23S rRNA internal transcribed spacer (ITS) sequevar analysis, all isolates of both species were classified within the Min-A ITS sequevar. Interestingly, four of the M. intracellulare isolates harbored IS1311, a M. avium-specific element. Two of three patients infected with "M. indicus pranii" had persistent positive sputum cultures after antibiotic therapy, indicating the clinical relevance of this study.

Conclusions: This analysis highlights the importance of precise identification of clinical isolates genetically close to Mycobacterium species, and suggests that greater attention should be paid to nontuberculous mycobacteria lung disease caused by " $M$. indicus pranii".

Keywords: Mycobacterium intracellulare, Mycobacterium indicus pranii, Multigene sequence-based typing, Insertion elements

\footnotetext{
* Correspondence: sjshin@yuhs.ac; wjkoh@skku.edu

${ }^{\dagger}$ Equal contributors

${ }^{3}$ Department of Microbiology, Institute for Immunology and Immunological Diseases, Brain Korea 21 PLUS Project for Medical Science, Yonsei University College of Medicine, Seoul, South Korea

'Division of Pulmonary and Critical Care Medicine, Department of Medicine, Samsung Medical Center, Sungkyunkwan University School of Medicine, Seoul, South Korea

Full list of author information is available at the end of the article
} 


\section{Background}

Mycobacterium avium complex (MAC) lung disease is the most common lung disease caused by nontuberculous mycobacteria (NTM) and its prevalence has been increasing worldwide [1-10]. MAC was originally composed of two species, M. avium and Mycobacterium intracellulare [11]. Many laboratories and studies reported these species as MAC because they are highly similar, and the clinical features of patients who are infected with these two species are considered indistinguishable $[11,12]$. However, some studies have suggested that differentiation between $M$. avium and $M$. intracellulare may have epidemiologic and clinical relevance $[13,14]$.

M. intracellulare is a major cause of MAC lung disease in many countries [2]. Molecular studies have revealed the presence of additional taxonomic groups or sequence variants (sequevars) that are closely related to M. intracellulare. Several new species were recently identified including Mycobacterium chimaera, Mycobacterium colombiense, Mycobacterium arosiense, Mycobacterium vulneris, Mycobacterium marseillense, Mycobacterium timonense, Mycobacterium bouchedurhonense, Mycobacterium mantenii, and Mycobacterium yongonense [15-20]. However, data regarding the proportion of these new species that are etiologic organisms in patients with previously diagnosed $M$. intracellulare lung disease are very limited.

The methods of correct identification for mycobacterial species in clinical laboratories have changed dramatically over the past two decades. Molecular methods have now surpassed biochemical tests and high-performance liquid chromatography as the method of choice for identifying NTM [21]. Polymerase chain reaction (PCR) restriction fragment length polymorphism analysis (PRA) based on restriction digestion products of specific genes such as hsp65, 16S rRNA, rpoB, and 16S-23S rRNA internal transcribed spacer (ITS) has been reported as a rapid, feasible, and inexpensive diagnostic method [22-25]. The gold standard identification method of $16 \mathrm{~S}$ rRNA gene sequencing and sequencing of each loci as a single identification target has failed to discriminate closely related Mycobacterium species such as MAC, Mycobacterium abscessus-Mycobacterium chelonae, Mycobacterium farcinogenes-Mycobacterium senegalense, Mycobacterium kansasii-Mycobacterium gastri, and Mycobacterium marinum-Mycobacterium ulcerans $[26,27]$. Recently, multigene sequence-based typing has been suggested as the new standard method for identifying Mycobacterium species that are not well discriminated by $16 \mathrm{~S}$ rRNA gene sequences alone [8, 28-30].

In our institution, the rpoB-PRA method had been used for species identification and diagnosis of MAC lung disease [14]. Recently published papers have emphasized the importance of taxonomy in distinguishing the many species and subspecies of MAC. Nonsequencing methods or $16 \mathrm{~S}$ rRNA sequencing might fail to distinguish closely related species [31, 32], indicating that nonsequencing-based approaches or analysis of a single target are not suitable for the accurate identification of (sub-)species belonging to MAC.

Thus, the aim of this study was to re-identify clinical isolates from patients previously diagnosed with $M$. intracellulare lung disease and to characterize their molecular pattern. For this purpose, the following methods were used: (1) multigene sequence-based typing of $16 \mathrm{~S}$ rRNA, rpoB, hsp65 and ITS genes, (2) hsp65 and ITS sequevar-based classification, and (3) insertion element analysis. Finally, three "Mycobacterium indicus pranii" strains that were previously identified as $M$. intracellulare were re-identified. The clinical characterization of lung disease caused by these three " $M$. indicus pranii" infections was described. " $M$. indicus pranii" is of specific interest due to its evolutionary significance and therapeutic potential in various disease processes. This study raises the possibility of " $M$. indicus pranii" as a pathogenic organism in the appropriate host and clinical situation, a notion not previously suggested in prior publications.

\section{Methods}

\section{Study subjects}

Clinical isolates from 77 consecutive patients who were newly diagnosed with $M$. intracellulare lung disease from Jan. 2008 to Dec. 2009 at Samsung Medical Center (a 1,961-bed referral hospital in Seoul, Korea) were collected and stored. This study was approved by the Institutional Review Board of Samsung Medical Center (File No. 200809-016). All patients' record and information was anonymized and de-identified prior to analysis. All patients met the diagnostic criteria for NTM lung disease [11]. All patients were immunocompetent and none of the patients tested positive for human immunodeficiency virus. Baseline patient characteristics are summarized in Table 1.

The isolates were collected before initiating antibiotic treatment for $M$. intracellulare lung disease. NTM species were identified as $M$. intracellulare by PRA based on the $r p o B$ gene at time of diagnosis [14].

\section{Identification of patient isolates by multigene sequence-based typing}

NTM were propagated in Middlebrook 7H9 broth (Difco Laboratories, Detroit, MI, USA) supplemented with $10 \%$ (vol/vol) oleic acid-albumin-dextrose-catalase (OADC; BD Diagnostics). Mycobacterial DNA was extracted using a DNeasy Blood and Tissue Kit according to the manufacturer's instructions (Qiagen, Valencia, CA). Multigene sequence-based typing including hsp65, rpoB, ITS and 16S rRNA fragments was carried out 
Table 1 Clinical characteristics of 77 patients with previously diagnosed $M$. intracellulare lung disease

\begin{tabular}{ll}
\hline & No. (\%) or median (IQR) \\
\hline Age, years & $64(55-72)$ \\
Female & $40(52)$ \\
Body mass index $\left(\mathrm{kg} / \mathrm{m}^{2}\right)$ & $20.0(17.7-21.5)$ \\
Non-smoker & $55(71)$ \\
Previous history of TB treatment & $43(56)$ \\
Positive sputum AFB smear & $39(51)$ \\
Type & \\
$\quad$ Nodular bronchiectatic form & $47(61)$ \\
$\quad$ Fibrocavitary form & $22(29)$ \\
$\quad$ Unclassifiable form & $8(10)$ \\
\hline IQR interquartile ranges, TB tuberculosis, AFB acid-fast bacilli
\end{tabular}

using PCR primer sets as described previously (Table 2). The PCR products of target genes were subjected to sequence analysis. hsp65 and ITS sequevar analysis were performed as previously described [33, 34]. The nucleotide sequences of these genes were compared with data reported by BLAST analysis (http://www.ncbi.nlm.nih.gov/) against sequences from $M$. intracellulare ATCC13950 ${ }^{\mathrm{T}}, M$. intracellulare ATCC15985, M. intracellulare MOTT-64, $M$. intracellulare MOTT-02, M. intracellulare MOTT36Y, and "M. indicus pranii" MTCC9506. For phylogenetic analysis, sequences were trimmed using the CLUSTAL-W multiple sequence alignment program [35]. Phylogenetic trees were obtained from DNA sequences utilizing the neighbor-joining method and Kimura's two parameter

Table 2 Primers used in this study

\begin{tabular}{lll}
\hline Target & Sequence (5' to 3') of paired primers & Reference \\
\hline 165 rRNA & AGA GTT TGA TCC TGG CTC AG & {$[54]$} \\
ITS & GTA TTA CCG CGG CTG CTG & \\
& TTG TAC ACA CCG CCC GTC & {$[34]$} \\
hsp65 & TCT CGA TGC CAA GGC ATC & \\
& AAC GTC GTC CTG GAG AAG AA & {$[55]$} \\
rpoB & GCC TTC TCC GGC TTG TC & \\
& GGC AAG GTC ACC CCG AAG GG & {$[27]$} \\
IS900 & AGC GGC TGC TGG GTG ATC ATC & \\
& TGG ACA ATG ACG GTT ACG GAG GTG G & {$[37]$} \\
IS901 & CGC AGA GGC TGC AAG TCG TGG & \\
& CGA CGA CAG GAG TAG CGG TAT GGC & {$[38]$} \\
IS1311 & CCG TGC TGC GAG TTG CTT GAT GAG & \\
& GCG TGA GGC TCT GTG GTG AA & {$[37]$} \\
DT1 & ATG ACG ACC GCT TGG GAG AC & \\
& CGT TGG CTG GCC ATT CAC GAA GGA GT & {$[37]$} \\
& GCT AGT TGG ATC GCG CCG AAC ACC GG & \\
\hline
\end{tabular}

distance correction model with 1000 bootstrap replications supported by MEGA 6.0 software [36].

\section{Insertion element analysis}

Multiplex PCR was performed to detect four target genes, IS900, IS901, IS1311 and DT1, using previously described methods [37, 38]. PCR product sizes of $398 \mathrm{bp}, 754 \mathrm{bp}, 608 \mathrm{bp}$, and $296 \mathrm{bp}$ corresponded to amplification of IS900, IS901, IS1311, and DT1 targets, respectively (data not shown). Amplification of only the DT1 gene indicated $M$. intracellulare. PCR products of insertion elements were sequenced and the existence of a specific insertion element in each strain was confirmed. DNA isolated from Mycobacterium abscessus ATCC19977, Mycobacterium tuberculosis H37Rv ATCC27294, and Mycobacterium gastri ATCC15754 were used as negative controls for each primer set in each PCR run.

\section{Results}

Re-identification of clinical isolates by multigene sequence-based typing

Isolates from 77 patients diagnosed with $M$. intracellulare lung disease were re-identified. Clinical isolates from 74 (96\%) patients were identified as $M$. intracellulare and those from three $(4 \%)$ patients were identified as " $M$. indicus pranii" using multiple gene sequencing analysis (Table 3). The $16 \mathrm{~S}$ rRNA and ITS sequences of " $M$. indicus pranii" isolates were identical to those of the " $M$. indicus pranii" type strain (GenBank accession no. CP002275) and the $M$. intracellulare type strain (GenBank accession nos. GQ153276 and CP003322, respectively). However, the $r p o B$ and nearly complete $h s p 65$ sequences (PCR with hsp65-sequevar primer sets) of " $M$. indicus pranii" isolates (isolate 01, 46 and 70) were only identical to those of the "M. indicus pranii" type strain (GenBank accession no. CP002275). They were $99.6 \%(708 / 711)$ and $99.8 \%$ $(1413 / 1416)$ similar to the $r p o B$ and $h s p 65$ sequences of the $M$. intracellulare type strain (GenBank accession nos. JQ411539 and DQ284774, respectively). The phylogenetic tree of all isolates with $M$. intracellulare and " $M$. indicus pranii" type strains is shown in Figs. 1, 2 and 3.

In all, the 77 isolates were classified to seven different hsp65 sequevars according to the method described by Turenne et al. [33]. Four of these sequevars were well recognized as $M$. intracellulare type and related strains, and 3 were newly identified in this study. The new sequevars were coded N4, N5 and N6 [followed by the code name given in the previous paper [39]. Ten single nucleotide polymorphisms (SNPs) excluding SNPs reported in the previous study were identified in this study (Table 4). The distribution of hsp65 sequevars in the 77 isolates is shown in Tables 3 and 4. In all, 74 $M$. intracellulare and three " $M$. indicus pranii" isolates were classified as the Min-A ITS sequevar. 
Table 3 Re-identification using multigene sequence-based typing, distribution of hsp65, ITS sequevar analysis, and insertion elements

\begin{tabular}{llll}
\hline & $\begin{array}{l}\text { M. } \\
\text { intracellulare } \\
\text { (MI) }\end{array}$ & $\begin{array}{l}\text { "M. indicus } \\
\text { pranil" (MIP) }\end{array}$ & $\begin{array}{l}\text { Comparison between } \\
\text { MI and MIP }\end{array}$ \\
\hline $\begin{array}{l}\text { Identification and } \\
\text { diagnosis by }\end{array}$ & & & \\
$\begin{array}{l}\text { Non-sequencing } \\
\text { method (PRA) }\end{array}$ & 77 & 0 & Identical \\
$\begin{array}{l}\text { Multigene } \\
\text { sequence-based } \\
\text { typing }\end{array}$ & 74 & 3 & Different \\
16S rRNA & & & \\
ITS & & & Identical \\
rpoB & & & Identical \\
hsp65 & & & Different \\
Molecular \\
characterization
\end{tabular}

PRA, PCR restriction fragment length polymorphism analysis, ITS internal transcribed spacer

${ }^{a}$ Two species were not distinguished by previously published $h s p 65$ code, but code N6 identified in this study was different between the two species

${ }^{\mathrm{b}} \mathrm{PCR}$ results of insertion element are indicated as positive(+) or negative(-)

${ }^{c}$ Four isolates identified as $M$. intracellulare were positive for IS1311

\section{Distribution of insertion elements between $M$. intracellulare and $M$. indicus pranii strains}

All isolates were negative for IS900 (considered diagnostic for $M$. avium subsp. paratuberculosis) as well as IS901 (considered diagnostic for M. avium subsp. avium), and positive for DT1 (considered diagnostic for $M$. intracellulare and $M$. avium subsp. avium). Interestingly, four (5\%) isolates identified as $M$. intracellulare were positive for IS1311 (considered diagnostic for all members of $M$. avium subspecies). The IS1311 sequences of four $M$. intracellulare isolates were identical to those of the M. avium insertion sequence IS1311 transposase gene (GenBank accession no. U16276), indicating that IS1311 might truly exist in some M. intracellulare strains.

\section{Clinical characteristics of three patients with $M$. indicus pranii lung disease}

Three patients were re-diagnosed as having " $M$. indicus pranii" lung disease (Table 5 and Fig. 4). Two patients received combination antibiotic therapy including clarithromycin, ethambutol, rifampin, and streptomycin. Three isolates from each patient were identified as " $M$. indicus pranii" using multigene sequence-based typing and had no mutations in $r r l$ (23S rRNA gene) according to sequencing analysis, which is known as main mechanism of acquired macrolide resistance in MAC [40]. Patient 1 died of an accident after five months of antibiotic therapy, and patient 2 showed persistent positive sputum cultures after 24 months of antibiotic therapy. Patient 3 was followed up without antibiotic treatment for 5.5 years because of mild symptoms.

\section{Discussion and conclusions}

In this study, clinical isolates from 77 patients that were previously diagnosed with $M$. intracellulare lung disease over a two-year period were re-identified. Species identification was initially performed by a non-sequencing method and then species were re-identified using a sequencing method. Among the 77 isolates identified as M. intracellulare by PRA at the time of diagnosis, 74 isolates were repeatedly identified as $M$. intracellulare. The remaining three were re-identified as " $M$. indicus pranii" by multigene sequence-based typing. However, hsp65 and ITS sequevar analyses were not precise enough to discriminate between $M$. intracellulare and "M. indicus pranii" in this study. To our knowledge, this is the first study to report documented cases of " $M$. indicus pranii" lung disease in humans.

$M$. intracellulare isolate 64 with code $\mathrm{N} 4$ and isolate 53 with code N5 were negative for IS900, IS901, and IS1311, and positive for DT1. Since classification among MAC subsets based on the hsp65 sequevar has been proposed [33], there have been several studies published on $M$. avium hsp65 sequevars, but none on $M$. intracellulare hsp 65 sequevars. The distribution of $M$. intracellulare hsp65 sequevars in other countries is unknown. However, two-thirds of the strains from this Koreanbased study were code 13 type, indicating that further studies to characterize this species are needed.

In general, IS1311 is present in all members of the $M$. avium subspecies and is not present in $M$. intracellulare. Four $M$. intracellulare isolates possessed identical IS1311 to that of the M. avium in this study, which is a novel observation. Since a number of different IS elements have been described in various NTM species, the 


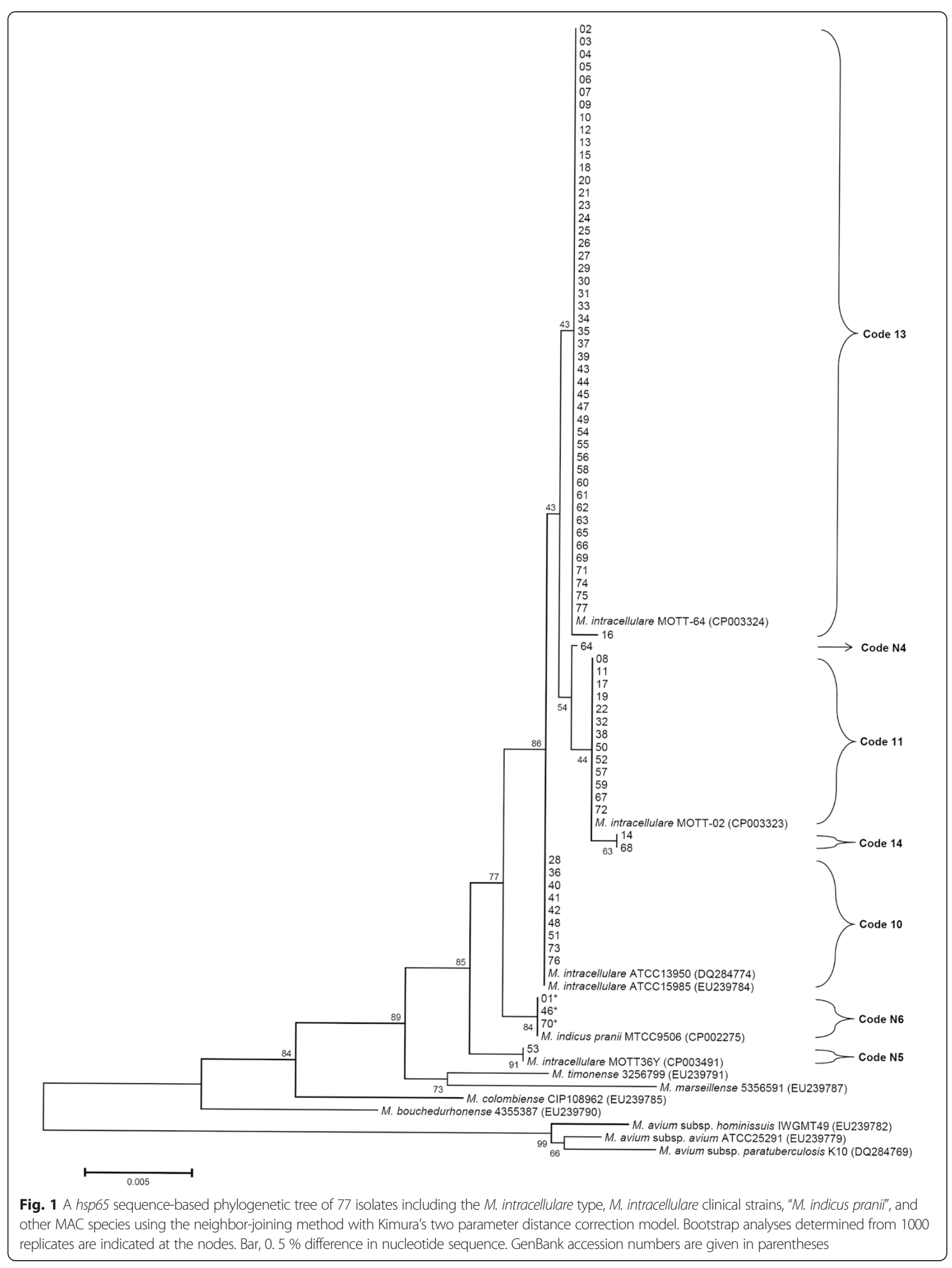




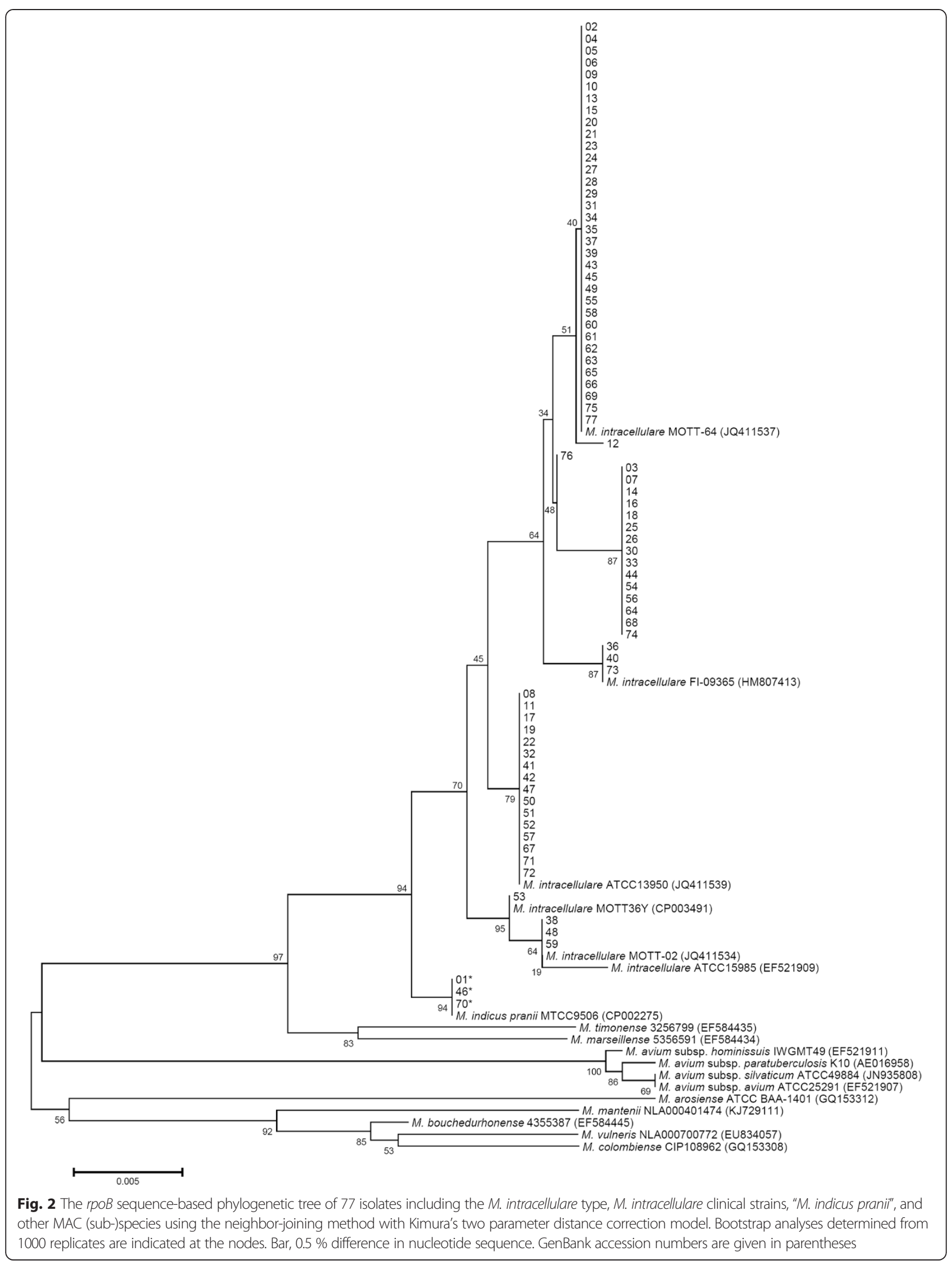




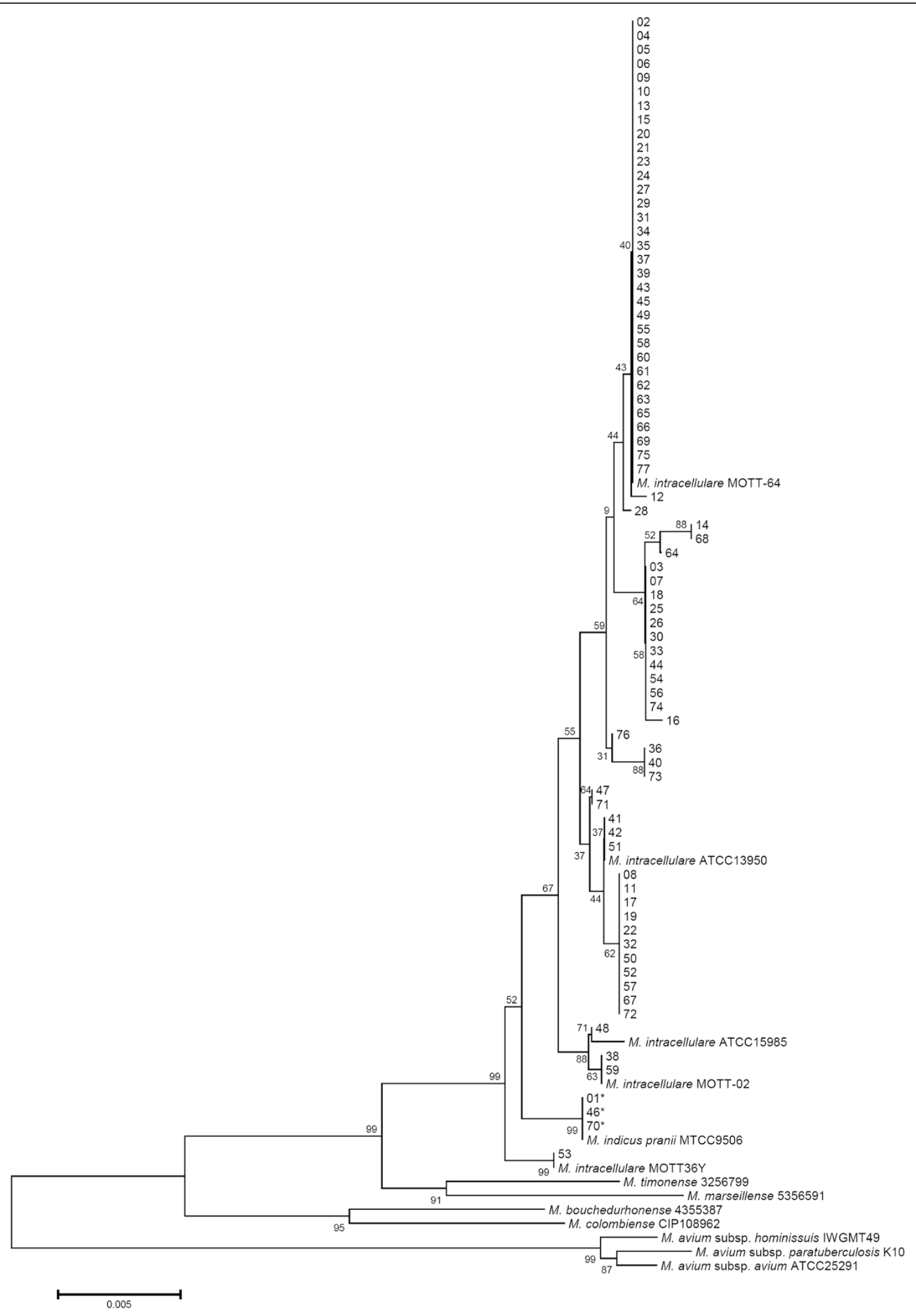

Fig. 3 The phylogenetic tree based on concatenated $h s p 65$ and rpoB sequences of 77 isolates including M. intracellulare type, M. intracellulare clinical strains, "M. indicus pranil", and other MAC (sub-)species using the neighbor-joining method with Kimura's two-parameter distance correction model. Bootstrap analyses determined from 1000 replicates are indicated at the nodes. Bar, $0.5 \%$ difference in nucleotide sequence. GenBank accession numbers are shown in Figs. 1 and 2 
Table 4 Identification of novel hsp65 sequevar codes and hsp65 SNPs among "M. indicus pranii" and M. intracellulare clinical strains compared to the $M$. intracellulare type strain

\begin{tabular}{|c|c|c|c|c|c|c|c|c|c|c|c|c|c|c|c|c|c|c|c|c|}
\hline \multirow{2}{*}{$\begin{array}{l}\text { hsp65 } \\
\text { code }^{a}\end{array}$} & \multirow[t]{2}{*}{ Species or strain } & \multicolumn{18}{|c|}{ Nucleotide at the indicated base pair position $(h s p 65)^{b}$} & \multirow{2}{*}{$\begin{array}{l}\text { No. of } \\
\text { isolates }\end{array}$} \\
\hline & & 192 & 198 & 249 & 279 & 285 & 459 & 477 & 555 & 633 & 726 & 804 & 921 & 933 & 1011 & 1191 & 1371 & 1423 & 1467 & \\
\hline $\begin{array}{l}\text { Code } \\
10\end{array}$ & $\begin{array}{l}\text { M. intracellulare } \\
\text { ATCC13950 }\end{array}$ & G & G & C & G & $\mathrm{T}$ & C & C & C & G & C & $C$ & C & $\mathrm{T}$ & G & G & G & C & C & 9 \\
\hline $\begin{array}{l}\text { Code } \\
11\end{array}$ & $\begin{array}{l}\text { M. intracellulare } \\
\text { FCC1804 }\end{array}$ & $\cdot$ & $\cdot$ & $\cdot$ & $\cdot$ & $\cdot$ & $\cdot$ & · & $\cdot$ & $\cdot$ & · & - & $\cdot$ & • & · & $\cdot$ & · & · & $\mathrm{T}$ & 13 \\
\hline $\begin{array}{l}\text { Code } \\
12\end{array}$ & M. intracellulare 96006 & • & • & • & • & • & $\mathrm{T}$ & • & · & - & - & - & • & - & • & • & - & • & • & 0 \\
\hline $\begin{array}{l}\text { Code } \\
13\end{array}$ & M. chimaera MI-JC & $\mathrm{T}$ & $\cdot$ & $\mathrm{T}$ & $\mathrm{T}$ & C & $\cdot$ & $\cdot$ & · & $\cdot$ & $\cdot$ & • & $\cdot$ & $\cdot$ & • & $\cdot$ & $\cdot$ & $\mathrm{T}$ & $\cdot$ & 48 \\
\hline $\begin{array}{l}\text { Code } \\
14\end{array}$ & M. intracellulare 90331 & · & · & · & - & • & $\cdot$ & · & . & · & · & - & · & · & · & · & A & • & $\mathrm{T}$ & 2 \\
\hline $\begin{array}{l}\text { Code } \\
\mathrm{N} 4^{c}\end{array}$ & $\begin{array}{l}\text { M. intracellulare clinical } \\
\text { isolate } 64\end{array}$ & $\mathrm{~T}$ & A & $\mathrm{T}$ & $\mathrm{T}$ & C & $\cdot$ & $\cdot$ & $\cdot$ & $\cdot$ & • & $\cdot$ & • & • & • & $\cdot$ & $\cdot$ & $\mathrm{T}$ & $\mathrm{T}$ & 1 \\
\hline $\begin{array}{l}\text { Code } \\
\mathrm{N}^{\mathrm{c}}\end{array}$ & $\begin{array}{l}\text { M. intracellulare } \\
\text { MOTT36Y }\end{array}$ & $\cdot$ & A & • & · & • & $\cdot$ & G & G & C & $\mathrm{T}$ & - & G & C & C & C & $\cdot$ & · & · & 1 \\
\hline $\begin{array}{l}\text { Code } \\
N 6^{c}\end{array}$ & $\begin{array}{l}\text { "M. indicus pranii" } \\
\text { MTCC9506 }\end{array}$ & $\cdot$ & $\cdot$ & $\cdot$ & $\cdot$ & • & $\cdot$ & • & $\cdot$ & $\cdot$ & $\cdot$ & G & • & $\cdot$ & C & C & $\cdot$ & $\cdot$ & $\cdot$ & 3 \\
\hline
\end{tabular}

${ }^{a}$ Classification according to Turenne et al. [33]

b. indicates the same base pair as in code 10; New base pair position found in this study are indicated by bold font

${ }^{\mathrm{c}} \mathrm{New}$ code types found in this study are designated by code N4, N5, and N6

species-specific IS elements have been revisited for MAC identification [37, 41, 42]. IS elements are mobile by nature, so there is a risk that similar elements are found in unrelated bacteria because of mobility to or from MAC organisms. Therefore, IS-based PCR differentiation of MAC must be performed in combination with other genetic analyses. Based on this study, DT1 is the optimal candidate marker gene for identification of $M$. intracellulare and " $M$. indicus pranii". Sequences analysis of $h s p 65$ and rpoB provides phylogenetic placement, allowing discrimination between the two species.

" $M$. indicus pranii" is initially named "Mycobacterium $w(M w)$ " and used as a potential leprosy vaccine [43]. The use of the name "Mycobacterium $w$ " gives an impression that $M w$ is related to the hypervirulent $M$. tuberculosis-W (Beijing strain) strain. To avoid confusion, Talwar et al. suggested using the nomenclature "Mycobacterium indicus pranii" [44]. However, neither of its name is found on the List of Prokaryotic Names with Standing in Nomenclature, and the designation "Mycobacterium indicus pranii" does not conform to the binomial naming convention used for bacterial species [45]. In a recent publication, Alexander et al. suggested that "M. indicus pranii" is a strain of $M$. intracellulare [46]. "M. indicus pranii" is considered to be a non-pathogenic microorganism and no human infections have been reported to date [47]. Use of the " $M$. indicus pranii" vaccine is based on the assumption that antigens shared between $M$. tuberculosis and this saprophytic mycobacterium is relevant for protective immunity and that " $M$. indicus pranii" lacks many of the harmful components present in $M$. tuberculosis [48]. "M. indicus pranii" immunotherapy did demonstrate protective efficacy against tuberculosis [49]. However, patients with pericardial tuberculosis who received " $M$. indicus pranii" injections demonstrated no significant benefit with respect to any reported outcomes in recent papers $[50,51]$. The efficacy of " $M$. indicus pranii" in severe sepsis has recently been reported [52].

On the basis of our findings, "M. indicus pranii" should be considered a cause of pulmonary disease in humans with pre-existing lung disease, such as tuberculosis and bronchiectasis. In addition, the virulence of

Table 5 Clinical characteristics of three patients with " $M$. indicus pranii" lung disease

\begin{tabular}{|c|c|c|c|}
\hline & Patient 1 & Patient 2 & Patient 3 \\
\hline Sex/Age & $M / 27$ & $\mathrm{~F} / 72$ & $M / 42$ \\
\hline $\begin{array}{l}\text { Previous TB } \\
\text { treatment }\end{array}$ & Yes & No & No \\
\hline $\begin{array}{l}\text { Sputum AFB } \\
\text { smear }\end{array}$ & Positive & Positive & Negative \\
\hline $\begin{array}{l}\text { Radiographic } \\
\text { type }\end{array}$ & Fibrocavitary & $\begin{array}{l}\text { Nodular } \\
\text { bronchiectatic }\end{array}$ & $\begin{array}{l}\text { Nodular } \\
\text { bronchiectatic }\end{array}$ \\
\hline Cavitary lesion & Bilateral & Unilateral & None \\
\hline $\begin{array}{l}\text { Clarithromycin } \\
\text { MIC ( } \mu \mathrm{g} / \mathrm{mL})\end{array}$ & 1.0 & 1.0 & $\leq 0.5$ \\
\hline $\begin{array}{l}\text { Antibiotic } \\
\text { treatment }\end{array}$ & Yes & Yes & No \\
\hline $\begin{array}{l}\text { Treatment } \\
\text { outcomes }\end{array}$ & $\begin{array}{l}\text { Death after } \\
5 \text { months of } \\
\text { treatment }\end{array}$ & $\begin{array}{l}\text { Persistent positive } \\
\text { sputum culture after } \\
24 \text { months of treatment }\end{array}$ & $\begin{array}{l}\text { Follow-up } \\
\text { without } \\
\text { treatment }\end{array}$ \\
\hline
\end{tabular}

TB tuberculosis, AFB acid-fast bacilli, MIC minimum inhibitory concentration 


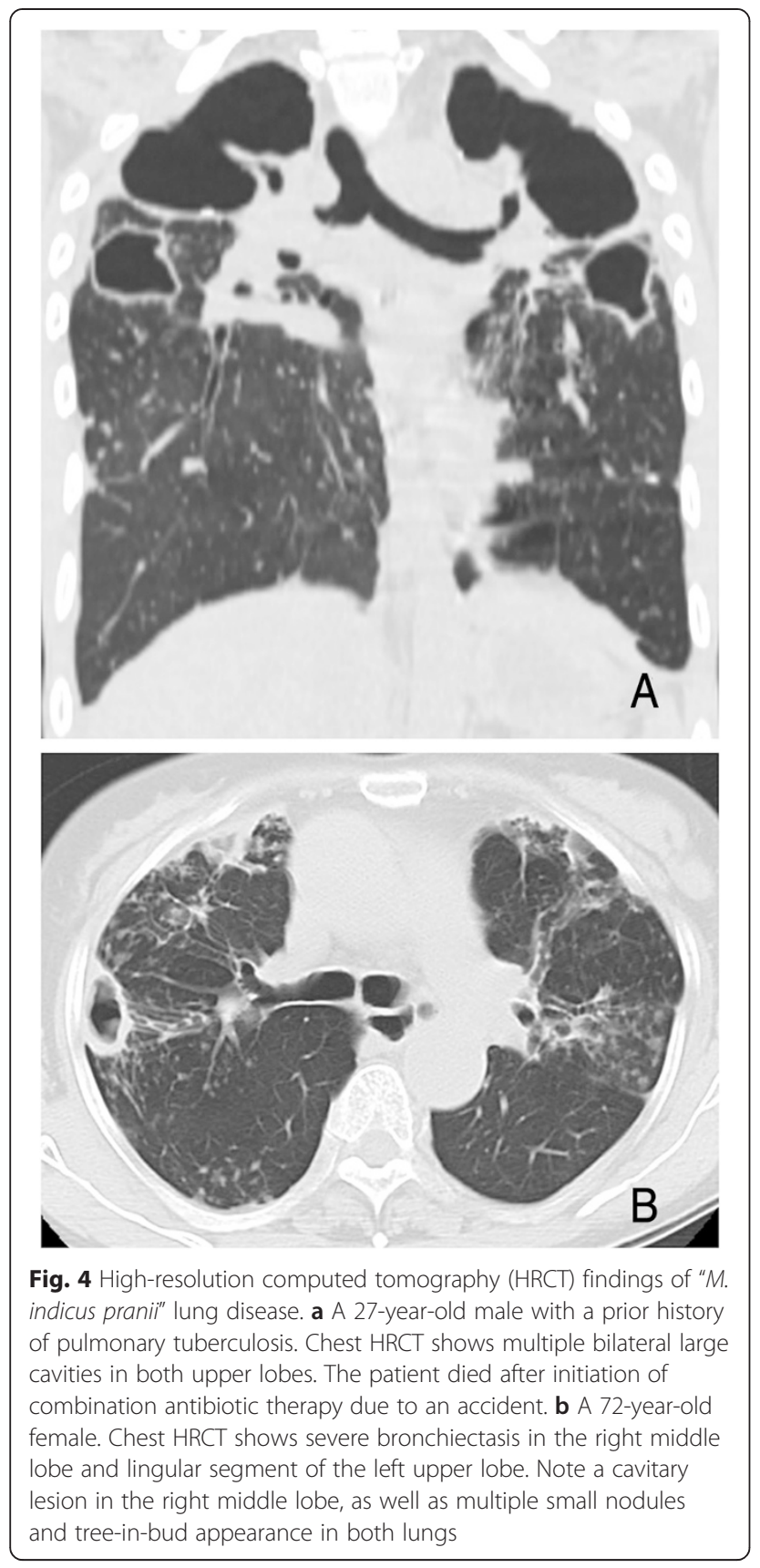

" $M$. indicus pranii" may vary according to geographical location. "M. indicus pranii" could be detected more frequently in the future as a consequence of increased genetic sequencing. Therefore, careful attention should be given to accurately identifying this Mycobacterium species. Further studies regarding the pathogenesis of " $M$. indicus pranii", including comparison with $M$. intracellulare, are needed.

Unlike M. tuberculosis, which has no environmental reservoir, NTM are ubiquitous microorganisms readily isolated from environmental sources, including soil and water. Despite the reportedly low virulence of NTM in immunocompetent human hosts, an increase in their isolation frequency has been seen in the last decade. Genetic analyses have greatly improved our understanding of the phylogeny and evolutionary diversity of NTM. Our study suggests that precise differentiation of $M$. intracellulare isolates may provide clinically relevant data including ecology, epidemiology, virulence, and treatment outcomes [32, 53].

The precise re-identification of clinical isolates initially identified as $M$. intracellulare by a non-sequencing method in patients with $M$. intracellulare lung disease revealed that most cases were caused by $M$. intracellulare. However, some were caused by " $M$. indicus pranii". Our study indicates the role of " $M$. indicus pranii" as an agent of severe and chronic lung disease in immunocompetent patients, suggesting that further study is needed to investigate its pathogenicity.

\section{Abbreviations}

MAC: Mycobacterium avium complex; NTM: nontuberculous mycobacteria; PCR: polymerase chain reaction; PRA: PCR restriction fragment length polymorphism analysis; ITS: internal transcribed spacer.

\section{Competing interests}

The authors declare that they have no competing interests.

\section{Authors' contributions}

SYK, HYP, SJS and WJK participated in the design of the study, analyzed and interpreted the data and wrote the manuscript. BHJ, KJ, HJH, CSK, and NYL participated in the design of the study, collected the data and helped to draft the manuscript. SJH performed experiments and contributed to revision of the manuscript. SJS and WJK conceived of the study, and participated in its design and coordination. All authors read and approved the final manuscript.

\section{Authors' information}

Not applicable.

\section{Availability of data and materials}

Not applicable.

\section{Acknowledgments}

This study was supported by a grant of the Korean Health Technology R\&D Project, Ministry for Health \& Welfare, Republic of Korea (A120647) and by the Basic Science Research Program through the National Research Foundation of Korea (NRF) funded by the Ministry of science, ICT \& Future Planning (NRF-2013R1A2A1A01009932).

\section{Funding}

The funding sources had no role in the design and conduct of the study; collection, management, analysis, and interpretation of the data; preparation, review, or approval of the manuscript; and decision to submit the manuscript for publication. The views expressed in this article are those of the authors and do not necessarily represent the views of the Korean Health Technology R\&D Project and NRF.

\section{Author details}

'Division of Pulmonary and Critical Care Medicine, Department of Medicine, Samsung Medical Center, Sungkyunkwan University School of Medicine, Seoul, South Korea. ${ }^{2}$ Department of Laboratory Medicine and Genetics, Samsung Medical Center, Sungkyunkwan University School of Medicine, Seoul, South Korea. ${ }^{3}$ Department of Microbiology, Institute for Immunology and Immunological Diseases, Brain Korea 21 PLUS Project for Medical Science, Yonsei University College of Medicine, Seoul, South Korea. 
Received: 27 January 2015 Accepted: 22 September 2015 Published online: 30 September 2015

\section{References}

1. Kendall BA, Winthrop KL. Update on the epidemiology of pulmonary nontuberculous mycobacterial infections. Semin Respir Crit Care Med. 2013:34:87-94.

2. Hoefsloot W, van Ingen J, Andrejak C, Angeby K, Bauriaud R, Bemer P, et al. The geographic diversity of nontuberculous mycobacteria isolated from pulmonary samples: an NTM-NET collaborative study. Eur Respir J. 2013;42:1604-13.

3. Ringshausen FC, Apel RM, Bange FC, de Roux A, Pletz MW, Rademacher J, et al. Burden and trends of hospitalisations associated with pulmonary non-tuberculous mycobacterial infections in Germany, 2005-2011. BMC Infect Dis. 2013;13:231.

4. Jankovic M, Samarzija M, Sabol I, Jakopovic M, Katalinic Jankovic V, Zmak L, et al. Geographical distribution and clinical relevance of non-tuberculous mycobacteria in Croatia. Int J Tuberc Lung Dis. 2013;17:836-41.

5. Marras TK, Mendelson D, Marchand-Austin A, May K, Jamieson FB. Pulmonary nontuberculous mycobacterial disease, Ontario, Canada, 1998-2010. Emerg Infect Dis. 2013;19:1889-91.

6. Russell CD, Claxton P, Doig C, Seagar AL, Rayner A, Laurenson IF. Nontuberculous mycobacteria: a retrospective review of Scottish isolates from 2000 to 2010. Thorax. 2014;69:593-5.

7. Koh WJ, Chang B, Jeong BH, Jeon K, Kim SY, Lee NY, et al. Increasing recovery of nontuberculous mycobacteria from respiratory specimens over a 10-year period in a tertiary referral hospital in South Korea. Tuberc Respir Dis (Seoul). 2013;75:199-204.

8. Jang MA, Koh WJ, Huh HJ, Kim SY, Jeon K, Ki CS, et al. Distribution of nontuberculous mycobacteria by multigene sequence-based typing and clinical significance of isolated strains. J Clin Microbiol. 2014;52:1207-12.

9. Chien JY, Lai CC, Sheng WH, Yu CJ, Hsueh PR. Pulmonary infection and colonization with nontuberculous mycobacteria, Taiwan, 2000-2012. Emerg Infect Dis. 2014;20:1382-5.

10. Wu J, Zhang Y, Li J, Lin S, Wang L, Jiang $Y$, et al. Increase in nontuberculous mycobacteria isolated in Shanghai, China: results from a population-based study. PLoS One. 2014;9:e109736.

11. Griffith DE, Aksamit T, Brown-Elliott BA, Catanzaro A, Daley C, Gordin F, et al. An official ATS/IDSA statement: diagnosis, treatment, and prevention of nontuberculous mycobacterial diseases. Am J Respir Crit Care Med. 2007;175:367-416.

12. Adjemian J, Olivier KN, Seitz AE, Holland SM, Prevots DR. Prevalence of nontuberculous mycobacterial lung disease in U.S. Medicare beneficiaries. Am J Respir Crit Care Med. 2012;185:881-6.

13. Han XY, Tarrand JJ, Infante R, Jacobson KL, Truong M. Clinical significance and epidemiologic analyses of Mycobacterium avium and Mycobacterium intracellulare among patients without AIDS. J Clin Microbiol. 2005;43:4407-12.

14. Koh WJ, Jeong BH, Jeon K, Lee NY, Lee KS, Woo SY, et al. Clinical significance of the differentiation between Mycobacterium avium and Mycobacterium intracellulare in M. avium complex lung disease. Chest. 2012;142:1482-8

15. Bang D, Herlin T, Stegger M, Andersen AB, Torkko P, Tortoli E, et al. Mycobacterium arosiense sp. nov., a slowly growing, scotochromogenic species causing osteomyelitis in an immunocompromised child. Int J Syst Evol Microbiol. 2008;58:2398-402.

16. Ben Salah I, Cayrou C, Raoult D, Drancourt M. Mycobacterium marseillense sp. nov., Mycobacterium timonense sp. nov. and Mycobacterium bouchedurhonense sp. nov., members of the Mycobacterium avium complex. Int J Syst Evol Microbiol. 2009;59:2803-8.

17. Kim BJ, Math RK, Jeon CO, Yu HK, Park YG, Kook YH, et al. Mycobacterium yongonense sp. nov., a slow-growing non-chromogenic species closely related to Mycobacterium intracellulare. Int J Syst Evol Microbiol. 2013;63:192-9.

18. Murcia MI, Tortoli E, Menendez MC, Palenque E, Garcia MJ. Mycobacterium colombiense sp. nov., a novel member of the Mycobacterium avium complex and description of MAC-X as a new ITS genetic variant. Int J Syst Evol Microbiol. 2006;56:2049-54.

19. van Ingen J, Boeree MJ, Kosters K, Wieland A, Tortoli E, Dekhuijzen PN, et al. Proposal to elevate Mycobacterium avium complex ITS sequevar MACQ to Mycobacterium vulneris sp. nov. Int J Syst Evol Microbiol. 2009;59:2277-82.
20. van Ingen J, Lindeboom JA, Hartwig NG, de Zwaan R, Tortoli E, Dekhuijzen PN, et al. Mycobacterium mantenii sp. nov., a pathogenic, slowly growing, scotochromogenic species. Int J Syst Evol Microbiol. 2009;59:2782-7.

21. van Ingen J. Diagnosis of nontuberculous mycobacterial infections. Semin Respir Crit Care Med. 2013;34:103-9.

22. Telenti A, Marchesi F, Balz M, Bally F, Bottger EC, Bodmer T. Rapid identification of mycobacteria to the species level by polymerase chain reaction and restriction enzyme analysis. J Clin Microbiol. 1993;31:175-8.

23. Domenech P, Menendez MC, Garcia MJ. Restriction fragment length polymorphisms of $16 \mathrm{~S}$ rRNA genes in the differentiation of fast-growing mycobacterial species. FEMS Microbiol Lett. 1994;116:19-24.

24. Roth A, Reischl U, Streubel A, Naumann L, Kroppenstedt RM, Habicht M, et al. Novel diagnostic algorithm for identification of mycobacteria using genus-specific amplification of the 16S-23S rRNA gene spacer and restriction endonucleases. J Clin Microbiol. 2000;38:1094-104.

25. Lee H, Park HJ, Cho SN, Bai GH, Kim SJ. Species identification of mycobacteria by PCR-restriction fragment length polymorphism of the rpoB gene. J Clin Microbiol. 2000;38:2966-71.

26. Rogall T, Wolters J, Flohr T, Bottger EC. Towards a phylogeny and definition of species at the molecular level within the genus Mycobacterium. Int J Syst Bacteriol. 1990:40:323-30

27. Adekambi T, Colson P, Drancourt M. rpoB-based identification of nonpigmented and late-pigmenting rapidly growing mycobacteria. J Clin Microbiol. 2003:41:5699-708.

28. Dai J, Chen Y, Dean S, Morris JG, Salfinger M, Johnson JA. Multiple-genome comparison reveals new loci for Mycobacterium species identification. J Clin Microbiol. 2011:49:144-53.

29. Macheras E, Roux AL, Bastian S, Leao SC, Palaci M, Sivadon-Tardy V, et al. Multilocus sequence analysis and $r p o B$ sequencing of Mycobacterium abscessus (sensu lato) strains. J Clin Microbiol. 2011;49:491-9.

30. Hashemi-Shahraki A, Bostanabad SZ, Heidarieh P, Titov LP, Khosravi AD, Sheikhi N, et al. Species spectrum of nontuberculous mycobacteria isolated from suspected tuberculosis patients, identification by multi locus sequence analysis. Infect Genet Evol. 2013;20:312-24.

31. Schweickert B, Goldenberg O, Richter E, Gobel UB, Petrich A, Buchholz P, et al. Occurrence and clinical relevance of Mycobacterium chimaera sp. nov., Germany. Emerg Infect Dis. 2008;14:1443-6.

32. Wallace Jr RJ, lakhiaeva E, Williams MD, Brown-Elliott BA, Vasireddy S, Vasireddy $R$, et al. Absence of Mycobacterium intracellulare and presence of Mycobacterium chimaera in household water and biofilm samples of patients in the United States with Mycobacterium avium complex respiratory disease. J Clin Microbiol. 2013;51:1747-52.

33. Turenne CY, Semret M, Cousins DV, Collins DM, Behr MA. Sequencing of hsp65 distinguishes among subsets of the Mycobacterium avium complex. J Clin Microbiol. 2006;44:433-40.

34. Frothingham $\mathrm{R}$, Wilson $\mathrm{KH}$. Sequence-based differentiation of strains in the Mycobacterium avium complex. J Bacteriol. 1993;175:2818-25.

35. Larkin MA, Blackshields G, Brown NP, Chenna R, McGettigan PA, McWilliam $\mathrm{H}$, et al. Clustal W and Clustal X version 2.0. Bioinformatics. 2007;23:2947-8.

36. Tamura K, Stecher G, Peterson D, Filipski A, Kumar S. MEGA6: Molecular Evolutionary Genetics Analysis version 6.0. Mol Biol Evol. 2013;30:2725-9.

37. Shin SJ, Lee BS, Koh WJ, Manning EJ, Anklam K, Sreevatsan S, et al. Efficient differentiation of Mycobacterium avium complex species and subspecies by use of five-target multiplex PCR. J Clin Microbiol. 2010;48:4057-62.

38. Tran QT, Han XY. Subspecies identification and significance of 257 clinical strains of Mycobacterium avium. J Clin Microbiol. 2014;52:1201-6.

39. Iwamoto T, Nakajima C, Nishiuchi Y, Kato T, Yoshida S, Nakanishi N, et al. Genetic diversity of Mycobacterium avium subsp. hominissuis strains isolated from humans, pigs, and human living environment. Infect Genet Evol. 2012;12:846-52

40. Meier A, Kirschner $P$, Springer B, Steingrube VA, Brown BA, Wallace Jr RJ, et al. Identification of mutations in $23 \mathrm{~S}$ rRNA gene of clarithromycin-resistant Mycobacterium intracellulare. Antimicrob Agents Chemother. 1994;38:381-4.

41. Bartos M, Hlozek P, Svastova P, Dvorska L, Bull T, Matlova L, et al. Identification of members of Mycobacterium avium species by Accu-Probes, serotyping, and single IS900, IS901, IS1245 and IS901-flanking region PCR with internal standards. J Microbiol Methods. 2006;64:333-45.

42. Johansen TB, Djonne B, Jensen MR, Olsen I. Distribution of IS1311 and IS1245 in Mycobacterium avium subspecies revisited. J Clin Microbiol. 2005;43:2500-2. 
43. Saini V, Raghuvanshi S, Talwar GP, Ahmed N, Khurana JP, Hasnain SE, et al. Polyphasic taxonomic analysis establishes Mycobacterium indicus pranii as a distinct species. PLoS One. 2009;4, e6263.

44. Talwar GP, Ahmed N, Saini V. The use of the name Mycobacterium $w$ for the leprosy immunotherapeutic bacillus creates confusion with M. tuberculosis-W (Beijing strain): a suggestion. Infect Genet Evol. 2008;8:100-1.

45. Tindall BJ, Kampfer P, Euzeby JP, Oren A. Valid publication of names of prokaryotes according to the rules of nomenclature: past history and current practice. Int J Syst Evol Microbiol. 2006;56:2715-20.

46. Alexander DC, Turenne CY. "Mycobacterium indicus pranii" is a strain of Mycobacterium intracellulare. MBio. 2015;6, e00013.

47. Groschel MI, Prabowo SA, Cardona PJ, Stanford JL, van der Werf TS. Therapeutic vaccines for tuberculosis-a systematic review. Vaccine. 2014;32:3162-8

48. Kaufmann SH. Tuberculosis vaccines: time to think about the next generation. Semin Immunol. 2013;25:172-81.

49. Gupta A, Ahmad FJ, Ahmad F, Gupta UD, Natarajan M, Katoch V, et al. Efficacy of Mycobacterium indicus pranii immunotherapy as an adjunct to chemotherapy for tuberculosis and underlying immune responses in the lung. PLoS One. 2012;7, e39215.

50. Mayosi BM, Ntsekhe M, Bosch J, Pandie S, Jung H, Gumedze F, et al. Prednisolone and Mycobacterium indicus pranii in tuberculous pericarditis. N Engl J Med. 2014;371:1121-30.

51. Chaisson RE, Post WS. Immunotherapy for tuberculous pericarditis. N Engl J Med. 2014;371:1155-7.

52. Sehgal IS, Agarwal $R$, Aggarwal $A N$, Jindal SK. A randomized trial of Mycobacterium w in severe sepsis. J Crit Care. 2015;30:85-9.

53. Frothingham $\mathrm{R}$, Wilson $\mathrm{KH}$. Molecular phylogeny of the Mycobacterium avium complex demonstrates clinically meaningful divisions. J Infect Dis. 1994;169:305-12.

54. Harmsen D, Dostal S, Roth A, Niemann S, Rothganger J, Sammeth M, et al. RIDOM: comprehensive and public sequence database for identification of Mycobacterium species. BMC Infect Dis. 2003:3:26

55. Ben Salah I, Adekambi T, Raoult D, Drancourt M. rpoB sequence-based identification of Mycobacterium avium complex species. Microbiology. 2008;154:3715-23.

\section{Submit your next manuscript to BioMed Central and take full advantage of:}

- Convenient online submission

- Thorough peer review

- No space constraints or color figure charges

- Immediate publication on acceptance

- Inclusion in PubMed, CAS, Scopus and Google Scholar

- Research which is freely available for redistribution 